\title{
Induction of high mobility group box 1 release from serotonin-stimulated human umbilical vein endothelial cells
}

\author{
KO-ICHI KAWAHARA ${ }^{1}$, TERUTO HASHIGUCHI ${ }^{1}$, KIYOSHI KIKUCHI ${ }^{1}$, SALUNYA TANCHAROEN ${ }^{2}$, \\ NAOKI MIURA $^{3}$, TAKASHI ITO ${ }^{1}$, YOKO OYAMA ${ }^{1}$, YUKO NAWA ${ }^{1}$, KAMAL K. BISWAS ${ }^{1}$, XIAOJIE MENG ${ }^{1}$, \\ YOKO MORIMOTO $^{4}$, BINITA SHRESTHA ${ }^{1}$, HISAYO SAMESHIMA ${ }^{1}$ and IKURO MARUYAMA ${ }^{1}$ \\ ${ }^{1}$ Department of Laboratory and Vascular Medicine, Cardiovascular and Respiratory Disorders, Advanced Therapeutics, \\ Kagoshima University Graduate School of Medical and Dental Sciences, Kagoshima 890-8520, Japan; ${ }^{2}$ Department of \\ Pharmacology, Faculty of Dentistry, Mahidol University, Bangkok 10400, Thailand; ${ }^{3}$ Veterinary Teaching Hospital \\ and Laboratory of Veterinary Diagnostic Imaging, Faculty of Agriculture, Kagoshima University, \\ Kagoshima 890-0065; ${ }^{4}$ Department of Periodontology, Kagoshima University Graduate School
} of Medical and Dental Sciences, Kagoshima 890-8544, Japan

Received May 2, 2008; Accepted July 14, 2008

DOI: 10.3892/ijmm_00000066

\begin{abstract}
High mobility group box 1 (HMGB1) is a nonhistone nuclear protein which is released from the nucleus of activated macrophages into the extracellular space in response to stimuli such as endotoxin or necrosis. The HMGB1 functions as a potent proinflammatory cytokine in the extracellular spaces. Recently, HMGB1 has been implicated in the progression of atherosclerosis. However, the association between HMGB 1 and the development of atherosclerosis is poorly understood. Therefore, we examined whether serotonin (5-HT), a key factor involved in the development of atherosclerosis, induced HMGB1 release in human umbilical vein endothelial cells (HUVECs). We found that 5-HT induced the release of HMGB1 but not of ERK1/2 and JNK from HUVECs via the 5-HT receptor (5-HT1B)/p38 mitogen-activated protein kinase (MAPK) signaling pathway. The p38MAPK inhibitor SB203580 and the 5-HT1B antagonist GR55526 markedly inhibited HMGB1 release from 5-HT-stimulated HUVECs. The vascular endothelial growth factor (VEGF) derived from activated macrophages in atherosclerotic lesions also plays an important role in the progression of atherosclerosis. We found that HMGB1 induced VEGF production in macrophage-like RAW264.7 cells. HMGB 1 induced the activation of p38MAPK, ERK1/2
\end{abstract}

Correspondence to: Dr Ikuro Maruyama, Department of Laboratory and Vascular Medicine, Cardiovascular and Respiratory Disorders, Advanced Therapeutics, Kagoshima University Graduate School of Medical and Dental Sciences, 8-35-1 Sakuragaoka, Kagoshima 890-8520, Japan

E-mail: rinken@m3.kufm.kagoshima-u.ac.jp

Key words: high mobility group box 1, p38 mitogen-activated protein kinase, atherosclerosis, angiogenesis and Akt. The PI3-kinase inhibitor LY294002 significantly inhibited VEGF production in HMGB1-stimulated macrophages, while other kinase inhibitors did not. These results suggest that HMGB1 release may contribute as a risk factor in the development and progression of atherosclerosis.

\section{Introduction}

Atherosclerosis is a chronic inflammatory disease and is also regarded as a progressive disease arising from a combination of endothelial dysfunction and inflammation (1). Vascular endothelial cells (ECs) play a major role in maintaining cardiovascular homeostasis and thus, in preserving good health. The EC monolayer lining the blood vessels constitutes a barrier between circulating blood and the vessel wall. Moreover, ECs secrete many mediators that regulate platelet aggregation, coagulation, fibrinolysis and vascular tone. In cases where the defense mechanisms elicited during cardiovascular events are insufficient to compensate for the adverse effects caused by the risk factors, ECs promote the development of atherosclerosis; this suggests that the presence of endothelial dysfunction may be predictive of adverse cardiovascular events.

Serotonin (5-HT) is an important neurotransmitter involved in the development of atherosclerosis (2). Platelets participate in the development of atherothrombosis: they aggregate at sites of vessel injury and secrete 5-HT, which is involved in several vascular phenomena such as thrombus formation and atherogenesis (2). 5-HT also induces the expression of tissue factors and plasminogen activator inhibitor-I in the ECs of rats and promotes the proliferation, migration, and contraction of vascular smooth muscle cells (VSMCs) (3-5). This suggests that 5-HT may contribute to the development of atherosclerosis.

High mobility group box 1 (HMGB1) protein has two distinct functions in cellular systems. In the nucleus, HMGB1 functions as an intracellular regulator of the transcription process and plays a crucial role in the maintenance 
of DNA functions (6). HMGB1 is released into the extracellular space during necrosis of all eukaryotic cells or via mitogen-activated protein kinase (MAPK) signaling pathways during macrophage responses to inflammatory stimuli such as endotoxin, tumor necrosis factor (TNF)- $\alpha$ and C-reactive protein (CRP) $(7-9,12,25)$. HMGB1 acts as a potent proinflammatory cytokine through a multiligand receptor for advanced glycation end products (RAGE), toll-like receptor (TLR)-2, and TLR-4 $(8,10)$. HMGB1 stimulates a number of cells to release cytokines such as TNF- $\alpha$, interleukin (IL)-6, and IL-1ß. This suggests that extracellular HMGB1 plays a critical role in the development of several inflammatory diseases such as sepsis, rheumatoid arthritis (RA), disseminated intravascular coagulation (DIC), and periodontitis and in xenotransplantation $(7,8,11,13,14)$. HMGB1 plays a role in the pathogenesis of plaque formation and in the progression of atherosclerotic lesions $(9,15,16)$. However, it is widely accepted that endothelial dysfunction occurs early during the development of atherosclerosis $(1,2,17)$. Nevertheless, the association between HMGB 1 and ECs is poorly understood.

Vascular endothelial growth factor (VEGF) is an angiogenic factor which plays an important role in the progression of atherosclerotic lesions. Its expression is upregulated not only under hypoxic conditions but also in the presence of transforming growth factor- $\beta$, angiotensin II, basic fibroblast growth factor (bFGF) and IL-1ß, which are expressed in newly formed atherosclerotic lesions. Activated macrophages play a central role in the development of atherosclerosis due to their ability to produce numerous cytokines and growth factors. A recent study revealed that angiogenesis and recanalization can be induced by VEGFs derived from activated macrophages, functioning in an autocrine or paracrine manner (18). HMGB1 is also an angiogenic factor because it induces bFGF via RAGE.

The present study aimed to investigate the effects of 5-HT on the secretion of HMGB 1 by human umbilical vein endothelial cells (HUVECs) and to identify the underlying intracellular signaling pathway involved in these effects. Furthermore, we also investigated whether HMGB1 induced VEGF production in macrophage-like RAW264.7 cells.

\section{Materials and methods}

Antibodies. The anti-HMGB1 antibody was obtained from Shino-Test Co. (Kanagawa, Japan). Anti-phosphorylated (p)ERK1/2, anti-p-p38MAPK, anti-p-Jun-N-terminal kinase (JNK), and anti- $\beta$-actin antibodies were purchased from Cell Signaling Technology (Beverly, MA).

Cell culture. HUVECs were purchased from Takara Biomedical (Shiga, Japan). They were cultured in endothelial basal medium 2 (EBM2) supplemented with growth factors (Takara Biomedical) and in fetal bovine serum (FBS, Takara Biomedical) supplemented with $100 \mathrm{U} / \mathrm{ml}$ penicillin and $100 \mu \mathrm{g} / \mathrm{ml}$ streptomycin. The cells were grown to $90 \%$ confluence, and further treatments were performed in EBM2 (Takara Biomedical).

Murine macrophage-like RAW264.7 cells were obtained from the American Type Cell Culture Collection (Manassas, VA) and were maintained in RPMI-1640 medium (Sigma,
St. Louis, MO) supplemented with $10 \%$ FBS and $2 \mathrm{mM}$ glutamine.

Stimulation of HUVECs with 5-HT. The cultured HUVECs ( $2 \times 10^{6}$ cells per $6-\mathrm{cm}$ dish) were starved for $2 \mathrm{~h}$ in a serumfree Opti-MEM-I medium (Invitrogen, Carlsbad, CA) and were stimulated with lipopolysaccharide (LPS)-free 5-HT (Sigma) in the medium. Following treatment, the HMGB1 levels in the culture media were analyzed by Western blot analysis.

Stimulation of RAW264.7 cells with MAPK and PI3-kinase inhibitors. Specific inhibitors of p38 MAPK (SB203580; Calbiochem, La Jolla, CA), MAP kinase (MEK1/2; U-0126, Calbiochem), and PI3 kinase (LY294002, Calbiochem) were used to evaluate the functional role of signaling pathways in 5-HT-induced HMGB1 release or HMGB1-induced VEGF production.

Western blot analysis. The HMGB1 levels in the culture supernatants were analyzed by Western blotting as described previously (12). In brief, each culture supernatant was incubated with $50 \mu \mathrm{l}$ heparin-Sepharose $6 \mathrm{~B}$ beads for $4 \mathrm{~h}$. The heparin beads were then washed with $10 \mathrm{mM}$ phosphate buffer (pH 7.0), mixed with $50 \mu 1$ sample buffer [62.5 mM Tris- $\mathrm{HCl}$ (pH 6.8), $2 \%$ sodium dodecyl sulfate (SDS), $10 \%$ glycerol, and $0.002 \%$ bromophenol blue], and boiled for $5 \mathrm{~min}$. These HMGB1 samples $(40 \mu \mathrm{l})$ were subjected to $12 \%$ SDS-polyacrylamide gel electrophoresis (SDS-PAGE), and the separated proteins were subsequently transferred onto a nitrocellulose membrane (GE Healthcare Bio-Sciences KK, Piscataway, NJ). The membrane was blocked with 5\% nonfat dry milk in Tris-buffered saline (TBS, $\mathrm{pH}$ 7.4) containing $0.02 \%$ Tween-20 (TBST) at room temperature (RT) for $1 \mathrm{~h}$ and was then incubated with $2 \mu \mathrm{g} / \mathrm{ml}$ of the anti-HMGB1 antibody (Shino-Test) in TBST containing 1\% non-fat dry milk at RT for $3 \mathrm{~h}$. The membrane was washed and incubated with a horseradish peroxidase (HRP)-conjugated anti-rabbit IgG polyclonal antibody (Invitrogen) diluted 1:3000 in TBST containing $2.5 \%$ non-fat dry milk at RT for $1 \mathrm{~h}$. The membrane was washed once more, and the immunoreactive bands formed were visualized using the enhanced chemiluminescence (ECL) detection system (GE Healthcare BioSciences KK).

Immunofluorescence microscopy. Immunofluorescence microscopy was performed as described previously (12). In brief, $5 \times 10^{5}$ HUVECs per well were cultured on 4-well BioCoat Collagen I culture slides (BD Biosciences, San Jose, CA). Following HUVEC stimulation as described above, the slides were washed with phosphate-buffered saline (PBS) and fixed with the OptiLyse $\mathrm{C}$ reagent (BD Biosciences) containing $0.1 \%$ Triton $\mathrm{X}-100$ (Sigma). The slides were blocked with $1 \%$ bovine serum albumin (BSA) in PBS containing $0.1 \%$ Triton-X 100 (PBST) for $1 \mathrm{~h}$, incubated with $1 \mu \mathrm{g} / \mathrm{ml}$ of the rabbit anti-HMGB1 antibody at RT for $1 \mathrm{~h}$, and then washed with PBST. Further, they were incubated with fluorescein isothiocyanate (FITC)-labeled goat antirabbit IgG (Invitrogen) for $1 \mathrm{~h}$, washed with PBST, and finally labeled with 4',6-diamidino-2-phenylindole (DAPI; 
A

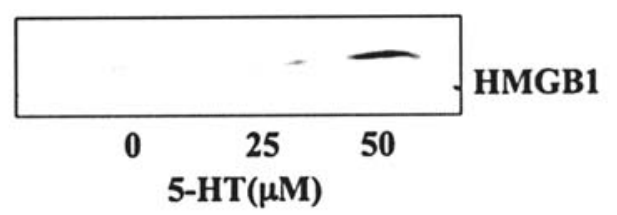

B

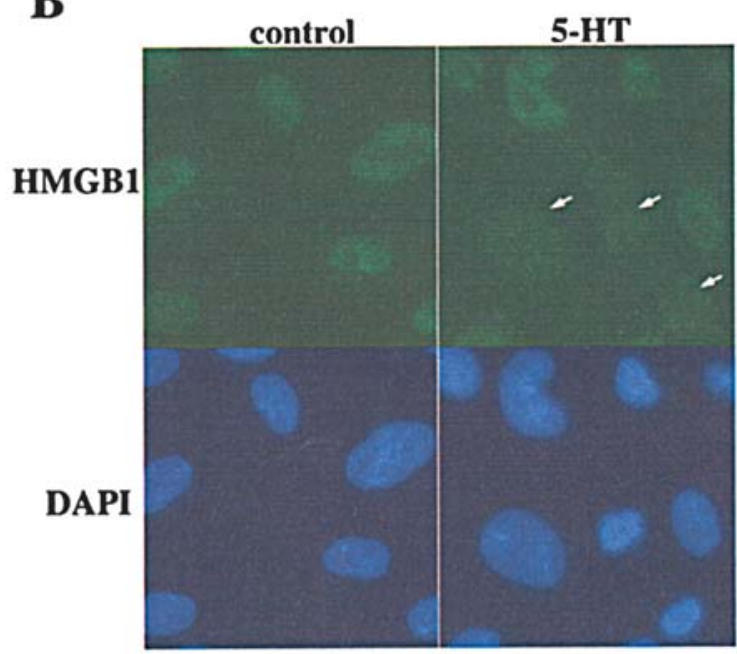

C

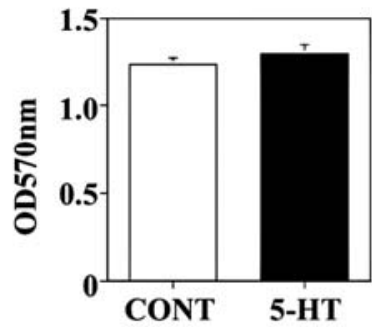

D

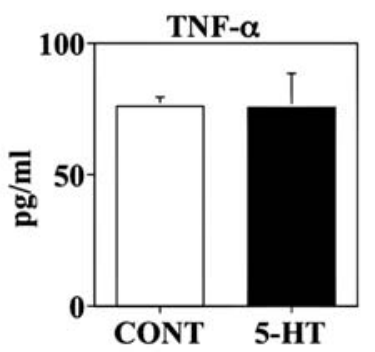

Figure 1. HMGB1 release from 5-HT-stimulated HUVECs. (A) Dosedependent effect of 5-HT in HUVECs. HUVECs were incubated with 5-HT $(0,25$, or $50 \mu \mathrm{M})$ for $16 \mathrm{~h}$. The HMGB1 levels were analyzed by Western blotting. (B) Translocation of HMGB1 from the nucleus into the extracellular space in response to 5-HT treatment. HUVECs were incubated in the absence or presence of 5-HT $(50 \mu \mathrm{M})$ for $16 \mathrm{~h}$. The fixed cells were incubated with rabbit anti-HMGB1 polyclonal antibody, followed by incubation with FITC-labeled anti-rabbit IgG. The nuclei were labeled with DAPI. Original magnification, $x 400$. Arrows indicate the translocation of HMGB1 from the nucleus. (C) Effects of 5-HT on cell viability. HUVECs were incubated with 5 -HT $(50 \mu \mathrm{M})$, and the cell viability was evaluated by an MTT assay. (D) Effects of 5-HT on TNF- $\alpha$ production. The HUVECs were treated with 5-HT $(50 \mu \mathrm{M})$ for $16 \mathrm{~h}$, following which the TNF- $\alpha$ levels in the culture supernatants were analyzed by performing ELISA. The values are presented as means \pm SD. The data shown are the representative values for 3 independent experiments. "Statistically significant $(\mathrm{P}<0.05)$ change.

Wako Chemicals, Japan) to visualize the cell nuclei. Finally, the slides were washed and examined under an Axioskop microscope (Carl Zeiss, Oberkochen, Germany).
MTT assay. Cell viability was analyzed by using the 3-(4,5dimethylthiazol-2-yl)-2,5-diphenyltetrazolium bromide (MTT) assay kit (Wako Chemicals), as described previously (13). In brief, the cells were cultured in 96-well plates $(100 \mu 1$ of medium per well) in the absence or presence of 5-HT $(50 \mu \mathrm{M})$ for $16 \mathrm{~h}$. Subsequently, the cells were incubated with MTT $(25 \mu \mathrm{g} / \mathrm{ml}$ per well) for $3 \mathrm{~h}$. The formazan product was solubilized by treatment with $100 \mu 1$ dimethyl sulfoxide for $16 \mathrm{~h}$. The dehydrogenase activity was expressed as the ratio between the absorbance at the test wavelength of $570 \mathrm{~nm}$ and that at the reference wavelength of $630 \mathrm{~nm}$.

Enzyme-linked immunosorbant assay. The VEGF and TNF- $\alpha$ levels in the culture supernatants were determined by using a commercial enzyme-linked immunosorbant assay (ELISA) kit specific for mouse VEGF and TNF- $\alpha$ (R\&D Systems, $\mathrm{MN})$. All experiments were performed in triplicate.

RAGE small interfering RNA transfection analysis. RAGE small interfering RNA (siRNA) transfection was carried out as described previously (12). In brief, RAW264.7 cells $\left(8 \times 10^{5}\right.$ cells $\left./ \mathrm{ml}\right)$ were cultured in $6-\mathrm{cm}$ dishes for $24 \mathrm{~h}$; washed with the Opti-MEM-I medium; and transfected with RAGE siRNA $(20 \mu \mathrm{M})$ or control siRNA (Santa Cruz Biotechnology, Inc., Santa Cruz, CA) for 2 days, using Oligofectamine (Invitrogen). The transfected cells were treated with HMGB1 $(10 \mu \mathrm{g} / \mathrm{ml})$ for $20 \mathrm{~h}$, and the VEGF levels in the medium were analyzed by performing ELISA.

Statistical analysis. Statistical analysis was performed using the Student's t-test. Statistical significance was accepted at $\mathrm{P} \leq 0.05$.

\section{Results}

$H M G B 1$ release from 5-HT-stimulated HUVECs. The aim of this study was to determine whether 5-HT induced HMGB1 release from HUVECs. The HUVECs were incubated with 5-HT $(0-50 \mu \mathrm{M})$ for $16 \mathrm{~h}$, and the culture supernatant was analyzed by performing Western blotting with anti-HMGB1 antibodies. As shown in Fig. 1A, there was a 5-fold increase in the HMGB1 levels in the medium containing 5-HTstimulated HUVECs. We assessed whether HMGB1 was released from the HUVECs into the medium under the abovementioned conditions by performing microscopy. HUVECs were incubated in the absence or presence of 5-HT in 4-well slides for $16 \mathrm{~h}$. As shown in Fig. 1B, HMGB1 disappeared from the nuclei (arrows). Next, we examined whether 5-HT treatment decreased the viability of the HUVECs. The cells were incubated in the absence or presence of 5-HT for $16 \mathrm{~h}$, following which the MTT reagent was added to the medium. As shown in Fig. 1C, 5-HT did not affect cell viability. Since TNF- $\alpha$, which induces HMGB1 expression, stimulates the active pathway for HMGB1 release, we examined whether 5-HT induced TNF- $\alpha$ production in the HUVECs. As shown in Fig. 1D, 5-HT had no effect on TNF- $\alpha$ production in the HUVECs.

HMGB1 release from 5-HT-treated HUVECs via the p38MAPK pathway. Next, we examined whether the presence 
A

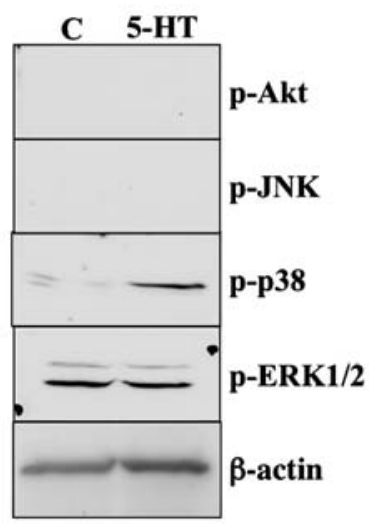

B

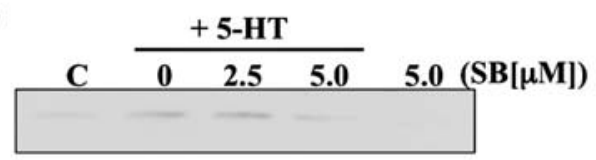

Figure 2. HMGB1 release in 5-HT-stimulated HUVECs via p38MAPK signaling. (A) 5-HT activates MAPKs. HUVECs were incubated with 5-HT $(50 \mu \mathrm{M})$ for $30 \mathrm{~min}$. The activation of p38MAPK, ERK1/2 and JNK was assayed by performing Western blot analysis with specific antibodies against p-p38MAPK, pERK1/2 and p-JNK1/2. (B) The HUVECs were pretreated with SB203580 $(0,2.5$, and $5 \mu \mathrm{M})$ for $15 \mathrm{~min}$, followed by incubation with 5-HT $(50 \mu \mathrm{M})$ for $16 \mathrm{~h}$. The HMGB1 levels in the culture supernatants were analyzed by using Western blotting. B-actin was used as the loading control. Each test was performed in 3 independent experiments.

\section{HMGB1}

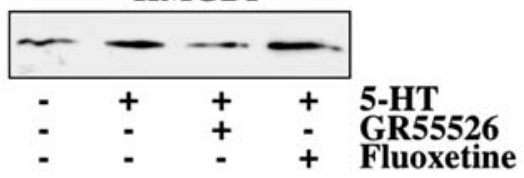

Figure 3. HMGB1 release via the 5-HT1B receptor in 5-HT-stimulated HUVECs. The HUVECs were incubated with GR55526 or fluoxetine for $2 \mathrm{~h}$, following which 5-HT was added to the medium and maintained for $16 \mathrm{~h}$. The HMGB1 levels were analyzed by Western blotting. Each test was performed in 3 independent experiments.

of 5-HT treatment activated MAPKs (ERK1/2, p38MAPK and JNK) or Akt in the HUVECs. The cells were incubated in the absence or presence of 5-HT for $30 \mathrm{~min}$, following which the cell lysates were analyzed by performing Western blotting with anti-p-ERK1/2, anti-p-p38MAPK, anti-pJNK, or anti-pAkt antibodies. As shown in Fig. 2A, 5-HT activated the phosphorylation of p38MAPK, but not the other kinases in the HUVECs. Moreover, we examined whether the 5-HTinduced HMGB1 release in the HUVECs was mediated via p38MAPK expression. The HUVECs were incubated with 5-HT alone or supplemented with SB203580 (0-5.0 $\mu \mathrm{M})$, which is a specific p38MAPK inhibitor, for $16 \mathrm{~h}$. As shown in Fig. 2B, SB203580 treatment markedly inhibited HMGB1 release in 5-HT-stimulated HUVECs.

Inhibition of HMGB1 release from the HUVECs following treatment with 5-HTIB antagonists. We examined whether treatment with a 5-HT receptor (5-HT1B) antagonist GR55526 or selective 5-HT reuptake inhibitors (SSRIs) such as fluoxetine inhibited HMGB1 release from 5-HT-stimulated HUVECs. HUVECs were preincubated with GR55526 or fluoxetine, following which 5-HT was added to the medium containing the HUVECs. After incubation for $16 \mathrm{~h}$, the HMGB1 levels in the medium were analyzed by Western blotting. As shown in Fig. 3, GR55526 but not fluoxetine markedly inhibited HMGB1 release.

VEGF induction in HMGB1-treated RAW264.7 cells. HMGB1 is reported to be an angiogenic factor that might be correlated with angiogenesis (19), which is an important process involved in the development of atherosclerosis. We hypothesized that HMGB 1 stimulates VEGF protein production in RAW264.7 cells. As shown in Fig. 4A, HMGB1 but not heat-treated HMGB1 significantly stimulated VEGF production in a dose-dependent manner. Further, HMGB1 treatment did not induce VEGF production in the RAGE knock-down cells. Moreover, the mechanism of VEGF production by HMGB 1 was found to be mediated via the Akt signaling pathway (Fig. 4B). Since RAGE is a specific HMGB1 receptor, we examined whether cells in which RAGE expression was knocked down by siRNA transfection produced VEGF following stimulation with HMGB1. As shown in Fig. 4C, under our experimental conditions, RAGE protein expression in the siRNA-treated cells was reduced to $75 \%$ as compared with the control cells $(100 \%)$.

\section{Discussion}

The present study is the first to demonstrate that 5-HT-treated HUVECs can actively and selectively release HMGB1 via the 5-HT1B receptor and that this release is specifically mediated by the p38MAPK signaling pathway. Furthermore, HMGB1 treatment triggered VEGF production via Akt signaling in RAW264.7 cells; this suggests that HMGB1 may play an important role in the development of atherosclerosis.

Platelet activation associated with EC dysfunction is the earliest event that occurs during the development of atherosclerosis. It is predicted that EC injury due to increased platelet activation causes HMGB1 release via a passive pathway. However, it is also possible that HMGB1 is released via an active pathway due to EC dysfunction. In fact, endotoxin is reported to induce HMGB1 release via an active pathway in ECs (17).

The present study shows that in HUVECs, 5-HT triggered HMGB1 release in an active rather than a passive manner. We believe that HMGB1 release triggered by $5-\mathrm{HT}$ treatment occurs via an active pathway. Our findings can be summarized as follows. First, under our experimental conditions, 5-HT treatment did not affect cell proliferation, cell death, and TNF- $\alpha$ production in the HUVECs. Second, treatment with the pharmacological inhibitor SB203580 but not U-0126 significantly inhibited HMGB1 release; this suggests that the activation of p38MAPK but not other kinases plays a crucial role in 5-HT-induced HMGB1 release. p38MAPK induces the production of key inflammatory mediators including TNF- $\alpha$ and IL-1ß, and HMGB1 which plays an important role in promoting inflammatory diseases $(12,20)$. Our findings 


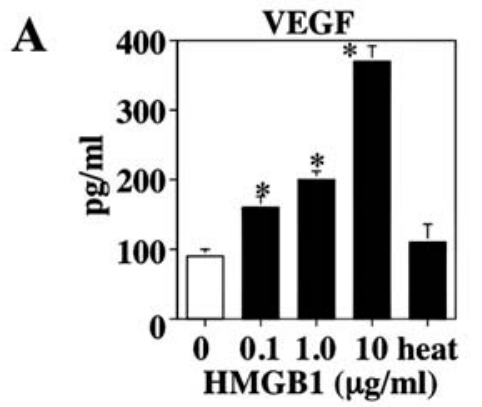

B

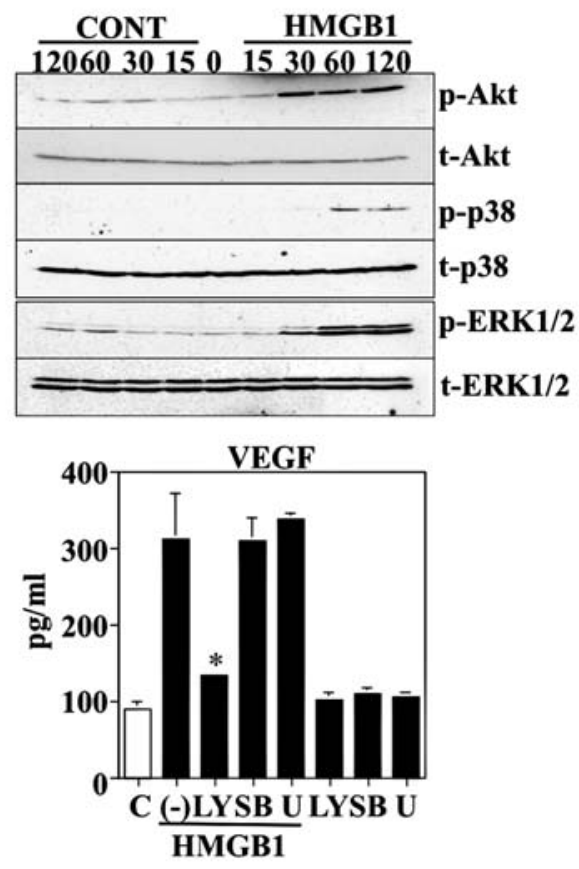

C

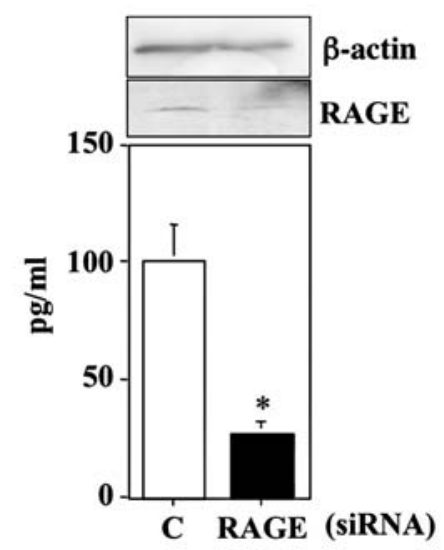

Figure 4. VEGF production in HMGB1-treated RAW264.7 cells. (A) HMGB1 induces VEGF production in a dose-dependent manner. The RAW264.7 cells were incubated in the absence or presence of HMGB1 $(0,0.1$, and $10 \mu \mathrm{g} /$ $\mathrm{ml}$ ) for $16 \mathrm{~h}$. The levels of VEGF protein released into the supernatants were analyzed by using ELISA. (B) VEGF expression following HMGB1-induced Akt activation. RAW264.7 cells were incubated with HMGB1 $(10 \mu \mathrm{g} / \mathrm{ml})$ for $30 \mathrm{~min}$. Activation of p38MAPK, ERK1/2, JNK and Akt was assayed by performing Western blot analysis with specific antibodies against pp38MAPK, pERK1/2, p-JNK1/2 and p-Akt. The RAW264.7 cells were pretreated with SB203580 $(5 \mu \mathrm{M})$, U-0126 $(5 \mu \mathrm{M})$, SP60025 $(5 \mu \mathrm{M})$ or LY294002 $(5 \mu \mathrm{M})$ for $15 \mathrm{~min}$, followed by incubation with HMGB1 $(10 \mu \mathrm{g} /$ $\mathrm{ml}$ ) for $16 \mathrm{~h}$. The levels of VEGF protein released into the supernatants were analyzed by using ELISA. (C) Inhibition of HMGB1-induced VEGF release by RAGE siRNA. RAW264.7 cells were transfected with control siRNA or RAGE siRNA, followed by incubation with HMGB1 $(10 \mu \mathrm{g} / \mathrm{ml})$ for $20 \mathrm{~h}$. The levels of VEGF in the supernatants and the expression of RAGE were analyzed by performing Western blotting with anti-RAGE antibody. B-actin was used as the loading control. The values are presented as means \pm SD. The data shown are the representative values for 3 independent experiments. * Statistically significant $(\mathrm{P}<0.05)$ change.

are consistent with those of a previous study which reported that HMGB1 release is mediated via the p38MAPK signaling pathway (12). Recent studies have suggested that HMGB1 release can occur through the activity of ERK1/2 or JNK rather than p38MAPK $(9,17)$. The discrepancy between these results and those of our study might be attributed to the differences in cell types and the stimulants examined. Third, HUVECs have 5-HT1B and transporter (21). In the HUVECs, the 5-HT-induced HMGB1 release was inhibited by the 5-HT1B antagonist GR55526, but not fluoxetine; this suggests that 5-HT directly triggers HMGB1 release via the active pathway in these cells.

Recent studies have demonstrated the presence and distribution of VEGF in the macrophages of atherosclerotic arteries (22). In non-atherosclerotic arteries, only weakly positive VEGF staining was observed in the intima of some sections and in the adventitia. In newly formed atherosclerotic lesions, VEGF staining was frequently observed in the subendothelial regions abundant in macrophages; this indicates that macrophages function as a potential source of VEGF (22).

We also demonstrated that HMGB1 induces VEGF mRNA expression (data not shown) and enhances VEGF protein levels in the culture supernatants of macrophage-like RAW264.7 cells via the Akt signaling pathway. Our data appear to support previous evidence in this regard $(22,23)$.
First, as mentioned above, HMGB1 treatment directly induces VEGF production in RAW264.7 cells but not in both HUVECs and VSMCs (data not shown). Second, to confirm the specificity of direct HMGB1 stimulation, we examined the effects of heat-treated HMGB1 and found that it did not induce VEGF production in RAW264.7 cells. Third, HMGB1 treatment did not induce VEGF production in cells wherein RAGE, a specific HMGB1 inhibitor, was knocked down. This suggests that HMGB1 directly induces VEGF production, mainly by macrophages. Fourth, VEGF production is mediated via the MAPK or Akt signaling pathway. Although in the inhibitor experiments, we found that HMGB1 activates both the MAPK and Akt signaling pathways, it mediates VEGF production via the Akt signaling pathway. Our data are consistent with a recent report stating that HMGB1 acts as a proangiogenic factor due to the induction of fibroblast growth factor (FGF), and that the inhibition of RAGE expression by siRNA eliminates VEGF production $(19,24)$. Thus, HMGB1 may contribute to the development of atherosclerosis by enhancing VEGF production.

To the best of our knowledge, this is the first study to demonstrate that 5-HT triggers active HMGB1 release via the 5-HT1B and p38MAPK signaling pathways in ECs. Our findings suggest that 5-HT may play an important role in inducing, amplifying, and prolonging the inflammatory processes, including atherosclerosis, by inducing the release of 
a key inflammatory mediator, HMGB1. Moreover, HMGB1 treatment induced VEGF production via the RAGE and Akt signaling pathways in RAW264.7 cells. This suggests that cross-talk between 5-HT and HMGB1 may be involved in the development and progression of atherosclerosis.

\section{Acknowledgements}

We thank Nobue Uto, Tomomi Morizono and Tomoka Nagasato for their excellent technical assistance. This study was supported by research grants from the Ministry of Education, Culture, Sports, Science and Technology of Japan, by Grants-in-Aid nos. 19791618 (Y.M.) and 18791341 (T.I.), and by a Health and Labor Sciences Research Grant from the Ministry of Health, Labor and Welfare (I.M.).

\section{References}

1. Ross R: Atherosclerosis - an inflammatory disease. N Engl J Med 340: 115-126, 1999.

2. Golino P, Crea F and Willerson JT: How to study the effects of platelet aggregation and thrombosis on coronary vasomotion and their clinical relevance. Ital Heart J 3: 220-225, 2002.

3. Nemecek GM, Coughlin SR, Handley DA and Moskowitz MA: Stimulation of aortic smooth muscle cell mitogenesis by serotonin. Proc Natl Acad Sci USA 83: 674-678, 1986.

4. Liu Y, Suzuki YJ, Day RM and Fanburg BL: Rho kinaseinduced nuclear translocation of ERK1/ERK2 in smooth muscle cell mitogenesis caused by serotonin. Circ Res 95: 579-586, 2004.

5. Kawano H, Tsuji H, Nishimura H, et al: Serotonin induces the expression of tissue factor and plasminogen activator inhibitor-1 in cultured rat aortic endothelial cells. Blood 97: 1697-1702, 2001.

6. Lu J, Kobayashi $\mathrm{R}$ and Brill SJ: Characterization of a high mobility group 1/2 homolog in yeast. J Biol Chem 271: 33678-33685, 1996.

7. Wang H, Bloom O, Zhang M, et al: HMG-1 as a late mediator of endotoxin lethality in mice. Science 285: 248-251, 1999.

8. Taniguchi N, Kawahara K, Yone K, et al: High mobility group box chromosomal protein 1 plays a role in the pathogenesis of rheumatoid arthritis as a novel cytokine. Arthritis Rheum 48: 971-981, 2003.

9. Kalinina N, Agrotis A, Antropova Y, et al: Increased expression of the DNA-binding cytokine HMGB1 in human atherosclerotic lesions: role of activated macrophages and cytokines. Arterioscler Thromb Vasc Biol 24: 2320-2325, 2004.

10. Park JS, Svetkauskaite D, He Q, Kim JY, Strassheim D, Ishizaka $A$ and Abraham E: Involvement of toll-like receptors 2 and 4 in cellular activation by high mobility group box 1 protein. J Biol Chem 279: 7370-7377, 2004.
11. Ito $\mathrm{T}$, Kawahara $\mathrm{K}$, Nakamura $\mathrm{T}$, et al: High-mobility group box 1 protein promotes development of microvascular thrombosis in rats. J Thromb Haemost 5: 109-116, 2007.

12. Kawahara K, Kamal KB, Unoshima M, et al: CRP induces high mobility group box-1 protein release through a p38 MAPK in the macrophage cell line RAW264.7 cells. Cardiovasc Pathol 17: 129-138, 2008.

13. Morimoto Y, Kawahara K, Tancharoen S, et al: Tumor necrosis factor- $\alpha$ stimulates gingival epithelial cells to release high mobility-group box 1. J Periodontal Res 43: 76-83, 2008.

14. Kawahara K, Setoyama K, Kikuchi K, et al: HMGB1 release in co-cultures of porcine endothelial and human $\mathrm{T}$ cells. Xenotransplantation 14: 636-641, 2007.

15. Inoue K, Kawahara K, Biswas KK, Ando K, Mitsudo K, Nobuyoshi $M$ and Maruyama I: HMGB1 expression by activated vascular smooth muscle cells in advanced human atherosclerosis plaques. Cardiovasc Pathol 16: 136-143, 2007.

16. Porto A, Palumbo R, Pieroni M, et al: Smooth muscle cells in human atherosclerotic plaques secrete and proliferate in response to high mobility group box 1 protein. FASEB J 20: 2565-2566, 2006

17. Treutiger CJ, Mullins GE, Johansson ASM, et al: High mobility group 1 B-box mediates activation of human endothelium. J Intern Med 254: 375-385, 2003.

18. Inoue $\mathrm{M}$, Itoh $\mathrm{H}$, Ueda $\mathrm{M}$, et al: Vascular endothelial growth factor (VEGF) expression in human coronary atherosclerotic lesions: possible pathophysiological significance of VEGF in progression of atherosclerosis. Circulation 98: 2108-2116, 1998 .

19. Mitola S, Belleri M, Urbinati C, et al: Cutting edge: extracellular high mobility group box-1 protein is a proangiogenic cytokine. J Immunol 176: 12-15, 2006.

20. Kuldo JM, Westra J, Asgeirsdóttir SA, et al: Differential effects of NF-\{kappa\}B and p38 MAPK inhibitors and combinations there of on TNF-\{alpha\}- and IL-1 \{beta $\}$-induced proinflammatory status of endothelial cells in vitro. Am J Physiol Cell Physiol 289: 1229-1239, 2005.

21. Ullmer C, Schmuck K, Kalkman HO and Lübbert H: Expression of serotonin receptor mRNAs in blood vessels. FEBS Lett 370: 215-221, 1995.

22. Ramos MA, Kuzuya M, Esaki T, et al: Induction of macrophage VEGF in response to oxidized LDL and VEGF accumulation in human atherosclerotic lesions. Arterioscler Thromb Vasc Biol 7: 1188-1196, 1998.

23. Salomonsson L, Svensson L, Pettersson S, Wiklund O and Ohlsson BG: Oxidised LDL decreases VEGFR-1 expression in human monocyte-derived macrophages. Atherosclerosis 169: 259-267, 2003.

24. Yamamoto Y, Yonekura H, Watanabe T, et al: Short-chain aldehyde-derived ligands for RAGE and their actions on endothelial cells. Diabetes Res Clin Pract 77: 30-40, 2007.

25. Scaffidi P, Misteli T and Bianchi ME: Release of chromatin protein HMGB1 by necrotic cells triggers inflammation. Nature 418: 191-195, 2002. 